\title{
QoS Parameters Investigations and Load Intensity Analysis, (A Case for Reengineered DCN)
}

\author{
${ }^{1}$ Udeze Chidiebele. C, 2Prof. H. C Inyiama \\ ${ }^{1,3}$ R \& D Department, Electronics Development Institute \\ (FMST-NASENI), Awka, Nigeria.
}

\begin{abstract}
This paper presents the simulation results on Reengineered DCN model considering Quality of Service (QoS) parameters in a homogeneous network for enterprise web application support. To make it feasible for the computer aided design (CAD) tool, a complete and robust real performance assessment of simulation scenarios is considered. This paper deals with a performance measurement and evaluation of a number of homogeneous end-to-end (e2e) DCN subnets taking into account a wide range of statistics. An active measurement approach of QoS parameters has been adopted for the studying of proprieties we called concise process model QoS. This paper validates the performance of the Reengineered DCN through simulations with MATLAB SimEvent which presents efficient performance metrics for its deployment. Considering the model hierarchy in context, the results shows that with the design model, a highly scalable DCN will be achieved.
\end{abstract}

Keywords-QoS; DCN; Model; CAD; Homogenous; Process component.

\section{INTRODUCTION}

Contemporarily, data centre networks (DCNs) have continued to attract a lot of interest in the enterprise networking market segments [1]. According to [2], a Data Centre is the consolidation point for provisioning multiple services that drive an enterprise business processes. A discrete event based process model of a reengineered data center communication network comprising of four data center LAN nodes with an inclusion of a virtualization server for creating virtual instances of resources and applications for DCN nodes was analyzed and presented in [3]. The perceived quality of TCP and UDP services on the reengineered DCN is primarily determined by parameters like packet loss, delay, jitter, throughput, and available bandwidth. For this purpose, several reference documents containing constraints regarding these parameters have been defined [4], [5], [6], and research have focused on the effects of these parameters on real time traffic (i.e. telephony) [7]. Understanding the statistics of QoS parameters is important for the appropriate design of DCN algorithms (routing, flow control, streaming), for the evaluation of network capability to support new value-added services (i.e. telephony, multimedia), for the study of network performance, for developing algorithms to detect security vulnerabilities and, finally, for the definition of Service Level Agreements (SLAs).

1. Why do packets drop in switched DCNs? And what are the effects in mission critical applications like: ERPs, Application Servers, E-commerce servers in DCN networks, etc.

\author{
3 Okafor Kennedy .C, 4Dr C. C. Okezie, \\ ${ }^{2,4}$ Electronics and Computer Engineering Department, \\ Nnamdi Azikiwe University, Awka, Nigeria.
}

2. How do we control traffic flow from access layer sources into switched data center networks without affecting network performance?

3. What is the behaviour of switch buffers at the congestion points, and reaction points under network oversubscription considering the various performance indices?

4. How do we achieve Agility in a Congested switched links for high performance computing?

A lot of works exists in literature that focused on the analysis of QoS parameters over different types of networks and protocols that runs on them [8], [9], [10], [11], [12]. Basically, by expanding distribution core and subnet backbone networks, performance parameters such as delay, utilization, bandwidth, and alongside the Carrier Sense Multiple Access with Collision Detection and Traffic Arbitration Message Protocol (CSMA/CD+TMAP) will create a stable and efficient DCN. This paper shall focus on metrics that will enhance performance in the reengineered DCN setup as shown in our system model in Fig.1.

\section{SySTEM DESIGN AND ASSUMPTIONS}

\section{A. System Model}

The high performance Reengineered DCN model configuration is shown in Fig. 1. It is assumed that the virtualization subnet is a composite powerful system for resource provisioning in the DCN. As discussed in [3], this paper develops a discrete event based process model and simulates a reengineered data center communication network comprising of four data center LAN nodes with an inclusion of a virtualization server for creating virtual instances of resources and applications for DCN nodes. A T-junction link modeled for 10GPs bandwidth was used. The MAC Controller block implements the CSMA/CD + TAMP (Carrier Sense Multiple Access with Collision Detection and Traffic Arbitration Message Protocol) Mechanism. This access technique of MAC bus system makes for an efficient and flexible data throughput while optimizing bandwidth in the reengineered DCN model. MATLAB SimEvent was employed in the model. Also, the CSMA/CD+ TAMP was modeled into the DCN MAC controllers for full duplex traffic flow while processing queues (frames) in such a manner as to avoid packet losses and congestion in the DCN when scalability consideration arises. Fig. 2 shows the process model architecture developed in this paper. This paper practically details the implementation 
methodology as well as discusses the System architecture mechanism in the section IV.

\section{B. Design Goals}

In designing our proposed DCN model, the main goals are to maintain throughput, low latency, fast convergence network and to create a cost effective network infrastructure that is based on robust power management infrastructure (SMART GRIDS), hence scalable, flexible with less administrative overhead.

The network should be capable of supporting high performance workloads (HPW) besides conventional ethernet traffic like cloud computing services. Meanwhile, multiple flows from various subnets are localized with VLAN strategies, but still shares link capacity fairly (buffer capacities). In the following, each of these goals is explained in details. The performance of today's DCN's (relevant to multimedia applications) is measured by QoS parameters such as:

1. Throughput or bandwidth

2. Delay or latency

3. Delay variation (delay jitter)

4. Loss or error rate

\section{Convergence/Stability}

From the application perspective, throughput refers to the data rate (bits per second) generated by the application. Throughput, measured in the number of bits per second, sometimes is called bit rate or bandwidth. Bandwidth is considered to be the network resource that needs to be properly managed and allocated to applications.

The throughput required by an application depends on the application characteristics. Fig. 1 shows the proposed system model developed in [3]. As shown in Fig 2, The process model architecture was implemented with MATLAB SimEvent. The top level model which includes four subnets DCN LAN nodes, each consisting of the following:

- An Application data packet block that models the DCN source data with its interface to a switch and router gateway.

- A MAC (medium access control) controller that governs the DCN's use of the shared channel and link resources.

- A T-junction that connects a LAN node to other DCN subnets networks.

- Switching Path Combiners, Gateway IP Clouds and Sinks.

The packet generation rate and packet size range are specified at the Application data packet blocks, the transmission buffer size specified at the MAC Controller blocks, and the length of the cable specified at the Cable blocks. The terminator, T-junction, and cable blocks at the bottom of the model represent physical components of the network.

\section{Experimental Testbed}

In this paper, to measure the system performance of the proposed Reengineered DCN infrastructure, MATLAB SimEvent [13] was used to achieve the set objective. We performed our experiments over the real test-bed described in Fig. 2. It is composed of a number of homogenous DCN networks which simplified the abstraction as depicted in Table 1. Over such test-bed, several configurations have been taken into account; indeed, the experiments have been performed by varying a number of configuration parameters like process model blocks, operating system, end user device, access network, transport protocol, and traffic condition. By combining all these variables we obtained about 20 different network conditions. The measurement stage has been performed in the time period between December 2012 and January 2012, in the simulation environment. For our proposed test bed, the infrastructure components include:

i. 4 DCN MAC controller subnets (80 DCN nodes) encapsulated in 4 subnets

ii. DCN Switch/Load balancer

iii. 4 Client Gateway for connection to the IP cloud

iv. Management Server with Virtualization support

v. Power management with SMART GRIDS

vi. Back office Applications (Network Monitoring, Configuration, Authentication, Authorization and Accounting ): Virtualization Management server for virtual instances, central network configuration \& Monitoring (Server 2008).

In Fig. 1, the Reengineered DCN model utilizes the infrastructure components outline above. A management server in subnet 3 has the role authenticating and monitoring the overall network for efficient service delivery as well as creating virtual instances for applications in the DCN. The DCN switch enables VLAN segmentation and propagates frames across the various subnets. Also, it interfaces the management virtual server, and DCN nodes, as shown in Fig. 2. The DCN LAN nodes were setup in their various subnets. The bridge switch is a speed redundancy layer supporting virtualization and load balancing. Access to the IP internet cloud is completed by the gateway and modem. After setting up the model, a simulation run was carried out to generate our graphical plots shown in this work (Fig. 3). Also, a consistency test carried out shows that the design model is stable and consistent before the simulation execution.

\section{DISCUSSION}

As for the active measurement procedure adopted in this work, we used the MATLAB SimEvent tool. By combining different pairs of process block set parameters, we generated a multitude of data for traffic patterns. In this way we generated controlled synthetic data that is realistic in the context of our proposal (Reengineered DCN). 

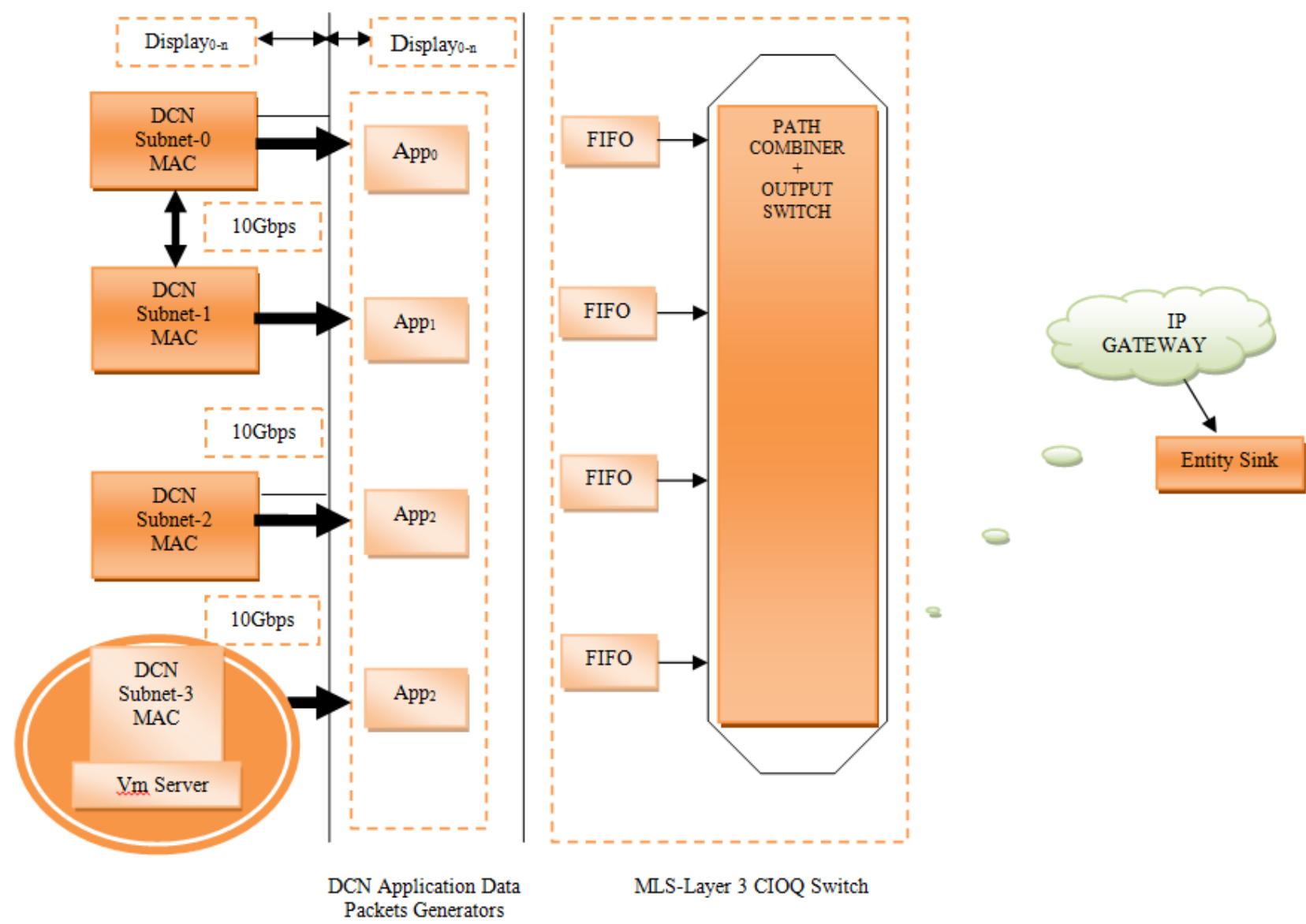

MLS-Layer 3 CIOQ Switch

Figure 1. The Process Model Architecture [3]

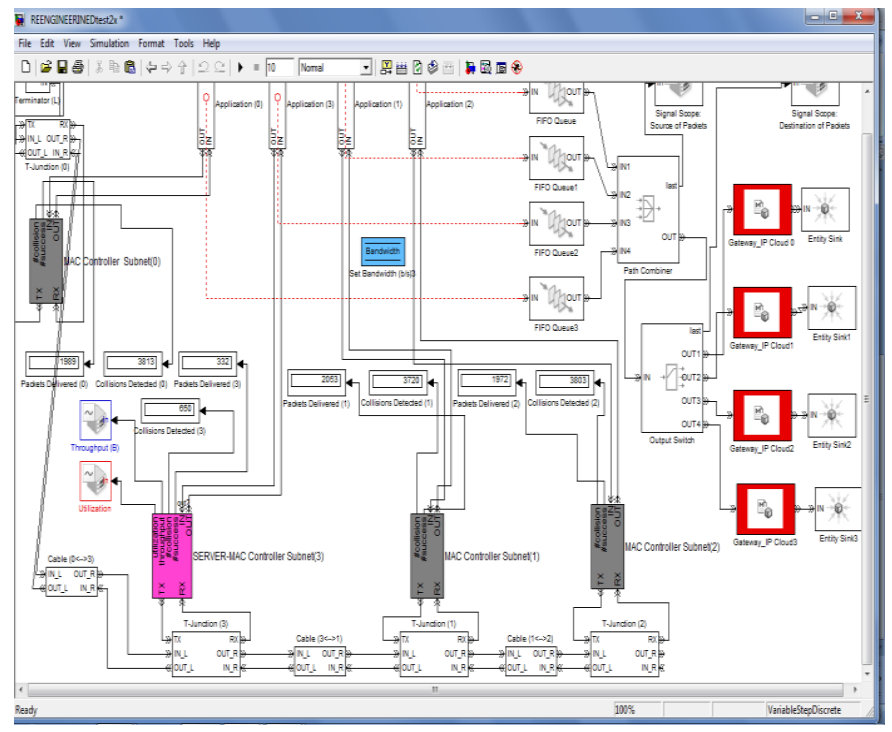

Figure 2. The SimEvent model

Also, in order to draw a reference curve for the parameter outlined in this paper, we considered only data traffic profile generated within our simulation environment. We would like to underline that our experiments have been carried out by using three traffic conditions namely Low, Medium, and High Traffic. For each of them a corresponding QoS is obtained, but interestingly our observation confirmed our model convergence and stability. Due to the nominal bandwidth used in our connections, we considered here only both high and low traffic condition for the communication pattern. We showed the behavior of throughput, latency, utilization, losses for our reengineered model measured over TCP connections.

Finally, it is worth noting that the presented results have been averaged on several tests in order to minimize the effect of random error on measures. Since there no changes in the QoS parameters with respect to variation in other parameters, we argue that our model is very stable.

The Reengineered DCN supports the following functionalities:

i. Parallel LAN and SAN infrastructure.

ii. Service oriented Architecture.

iii. Software as a service (SaaS), Platform as a service (PaaS)

iv. Server consolidation and Virtualization.

v. Dynamic Host Configuration Strategy (DHCS).

vi. Automatic Configuration.

vii. Bandwidth Control/Optimization. 
viii. Multiple Services.

ix. Enterprise Security Layout.

\section{SimUlation PARAMETERS}

In this section, we provide simulation results that support section III. We used MATLAB SimEvent [3] to generate the parameters for various case scenarios in the simulations. The model was configured for simulation using the traffic attributes as listed in Table 1.

TABLE I. PROCESS MODEL ATTRIBUTES

\begin{tabular}{|c|c|}
\hline \multicolumn{1}{|c|}{ Configurations } & Values \\
\hline $\begin{array}{l}\text { Simulation Duration for } \\
\text { Each Scenario }\end{array}$ & 1 Sec-15Secs \\
\hline Link Propagation Delays & 0.5 usecs \\
\hline Switch Output Buffer & $100-1400$ packets \\
\hline Simulation Seed & 128 \\
\hline Update Interval & 50000 Events \\
\hline Simulation Kernel & OS Optimized \\
\hline
\end{tabular}

By integrating established and well-known simulation strategies found in different works, we have set up a methodology and provided simulation result data for analysis from our validation tool. The data were captured via the SimEvent to "To Workspace block" which inputs a signal and writes the signal data to the MATLAB workspace [14]. The block writes the data to an array or structure that has the name specified by the block's variable name parameter. Table 2 shows the Re-DCN parameters used for the various load intensities in the process and network models.

\section{A. PERFORMANCE EVALUATION}

The Re-engineered DCN is a high performance model that is robust and scalable which is deemed fit for the enterprise domains. Having run the simulation in normal mode and collected the statistics for various cases, the figures below show their analysis. This paper used an aggregate scenario approach for homogeneous load intensities just by varying the key model parameters viz: Time, buffer sizes, and packet generation rates as depicted in Table 2a.To demonstrate the effects of these; we employed the MAC controller DCN simulation scenarios with the combinations of values for the above listed parameters. After running 20 simulations alongside varying the process model block parameters for our stipulated load intensities, the QoS parameters viz: Average throughput, latency, utilization stability and losses were computed for analysis. Table $2 \mathrm{~b}$ shows the packet delivered data and collision detected data without TAMP.

\section{B. SIMULATION RESULTS}

The process model developed with MATLAB Simevent was used to generate Fig. 3 to Fig. 15. Traffic characterization for the reengineered DCN gave the observed plot trends. From the process model, the subnet MAC Controller and FIFO Queues holds packets based on service policy of the DCN design hierarchy, hence the DCN switch is capable of determining the flow sequence in each of the incoming buffers at the beginning of service of each packet/frame arrival. The model behavior shows a workload support for enterprise wide deployments viz: cloud computing ERPs, content delivery network systems, and other services. Considering the various process model subnets, this work presents, a graphical plot analysis justifying our selected QoS.

In Fig. 3, packet flow in subnet 0,1,2, and 3 was observed to be greatly influenced by some forms of collisions which tend to affect data throughput in the regineered DCN model. This work introduced an enhance layer 2 protocol to suppress and enhance packet delivery in the reengineered DCN (subnet 0-3). Since the conventional ethernet networks works with CSMA/CD, traces of packet collisions in the subnet broadcast domains definitely affect the throughput response. Essentially, the Traffic arbitration message protocol (TAMP) strategy in the subnet MAC Controller block regulates efficient throughput by suppressing collision effects. Using linear regression concept in Table $2 \mathrm{~b}$, the $\mathrm{DCN}$ model residual value $\mathrm{R}(\mathrm{R}=$ 0.999), gives a significant index for the reengineered subnet 0 , 1, 2 and 3, hence guaranteed packet delivery. Figures 4, 5, 6 show that with the CSMA/CD+TAMP, the model will scale gracefully offering better performance than the conventional DCNs.

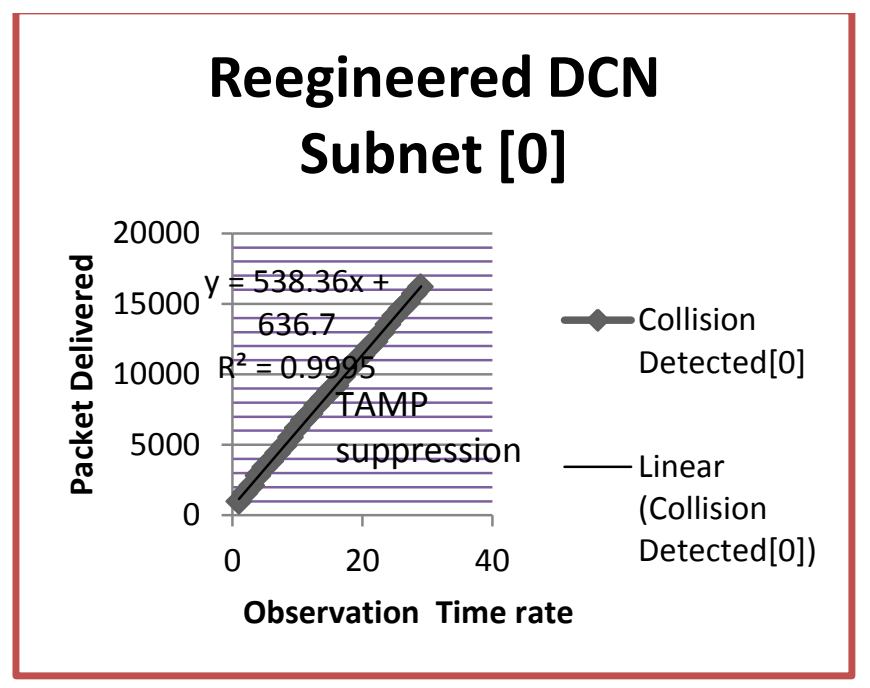

Figure 3. A plot of TAMP Suppression in Subnet [0] 
TABLE II. A: R-DCN SIMULATION PARAMETERS

\begin{tabular}{|c|c|c|c|c|c|c|c|c|}
\hline $\begin{array}{l}\text { Time } \\
\text { (Secs) }\end{array}$ & Buffer Sizes & MTUmax & $\begin{array}{l}\text { Packet } \\
\text { Generation } \\
\text { rate }\end{array}$ & $\begin{array}{l}\text { Average. } \\
\text { Throughput }\end{array}$ & $\begin{array}{l}\text { Average. } \\
\text { Latency }\end{array}$ & $\begin{array}{l}\text { Average. } \\
\text { Utilization }\end{array}$ & $\begin{array}{l}\text { Average. } \\
\text { Stability }\end{array}$ & Average. Losses \\
\hline 1 & 25 & 1500 & 100 & 111.4 & 107.4286 & 110.001818 & 109 & 110.83 \\
\hline 1.5 & 50 & 1500 & 150 & 130.2 & 100.125 & 105.1667 & 110.5 & 110.29 \\
\hline 2.0 & 100 & 1500 & 200 & 256 & 100.025 & 105.25 & 112 & 109.75 \\
\hline 2.5 & 150 & 1500 & 250 & 256.25 & 100.375 & 131.6888889 & 113.5 & 109.21 \\
\hline$\overline{3.0}$ & 200 & 1500 & 300 & 320 & 100.5 & 105.3333 & 115 & 108.67 \\
\hline 3.5 & 250 & 1500 & 350 & 320.6 & 100.625 & 141.6168889 & 116.5 & 108.13 \\
\hline 4.0 & 300 & 1500 & 400 & 355.5 & 100.75 & 105.4167 & 118 & 107.59 \\
\hline 4.5 & 350 & 1500 & 450 & 424 & 100.875 & 105.5 & 119.5 & 107.05 \\
\hline 5.0 & 400 & 1500 & 500 & 500 & 101 & 101.4866667 & 121 & 106.51 \\
\hline 5.5 & 450 & 1500 & 550 & 2179.6 & 101.125 & 105.5833 & 122.5 & 105.97 \\
\hline 6.0 & 500 & 1500 & 600 & 6081.8 & 94.3333 & 105.6667 & 124 & 105.43 \\
\hline 6.5 & 550 & 1500 & 650 & 6145.8 & 94.4444 & 110.1616889 & 125.5 & 104.89 \\
\hline 7.0 & 600 & 1500 & 700 & 6158.9 & 94.5556 & 105.75 & 127 & 104.35 \\
\hline$\overline{7.5}$ & 650 & 1500 & 750 & 6200.11 & 94.6667 & 100.6923 & 128.5 & 103.81 \\
\hline 8.0 & 700 & 1500 & 800 & 6240.5 & 94.7778 & 97.0714 & 130 & 103.27 \\
\hline 8.5 & 750 & 1500 & 850 & 6260 & 94.8889 & 100.8462 & 131.5 & 102.73 \\
\hline 9.0 & 800 & 1500 & 900 & 6239 & 95 & 100.9231 & 133 & 102.19 \\
\hline 9.5 & 850 & 1500 & 950 & 6300.5 & 95.1111 & 101 & 134.5 & 101.65 \\
\hline$\overline{10.0}$ & 900 & 1500 & 1000 & 6350.98 & 95.2222 & 101.0769 & 136 & 101.11 \\
\hline 10.5 & 950 & 1500 & 1050 & 6500 & 95.3333 & 101.1538 & 137.5 & 100.57 \\
\hline 11.0 & 1000 & 1500 & 1100 & 6800 & 94.4444 & 101.2308 & 139 & 100.03 \\
\hline 11.5 & 1050 & 1500 & 1150 & 7800 & 94.5556 & 101.3077 & 140.5 & 99.49 \\
\hline 12.0 & 1100 & 1500 & 1200 & 7950 & 94.6667 & 101.3846 & 142 & 98.95 \\
\hline 12.5 & 1150 & 1500 & 1250 & 7960 & 94.7778 & 100.7692 & 143.5 & 98.41 \\
\hline$\overline{13.0}$ & 1200 & 1500 & 1300 & 8000 & 94.8889 & 100.8462 & 145 & 97.87 \\
\hline$\overline{13.5}$ & 1250 & 1500 & 1350 & 8020 & 95 & 100.9231 & 146.5 & 97.33 \\
\hline 14.0 & 1300 & 1500 & 1400 & 8030 & 95.1111 & 101 & 148 & 96.79 \\
\hline 14.5 & 1350 & 1500 & 1450 & 8037 & 95.2222 & 101.0769 & 149.5 & 96.25 \\
\hline 15.0 & 1400 & 1500 & 1500 & 8046 & 95.3333 & 101.1538 & 151 & 95.71 \\
\hline
\end{tabular}


TABLE II. B: R-DCN TRAFFIC CHARACTERIZATION

\begin{tabular}{|c|c|c|c|c|c|c|c|c|}
\hline $\begin{array}{l}\text { Time } \\
\text { (Secs) }\end{array}$ & $\begin{array}{l}\text { Packet } \\
\text { Delivered[0] }\end{array}$ & $\begin{array}{l}\text { Collision } \\
\text { Detected[0] }\end{array}$ & $\begin{array}{l}\text { Packet } \\
\text { Delivered[1] }\end{array}$ & $\begin{array}{l}\text { Collision } \\
\text { Detected[1] }\end{array}$ & $\begin{array}{l}\text { Packet } \\
\text { Delivered[2] }\end{array}$ & $\begin{array}{l}\text { Collision } \\
\text { Detected[2] }\end{array}$ & $\begin{array}{l}\text { Packet } \\
\text { Delivered[3] }\end{array}$ & $\begin{array}{l}\text { Collision } \\
\text { Detected[3] }\end{array}$ \\
\hline 1 & 546 & 970 & 546 & 948 & 511 & 984 & 86 & 165 \\
\hline 1.5 & 882 & 1559 & 890 & 1524 & 862 & 1551 & 147 & 283 \\
\hline 2.0 & 1103 & 2086 & 1115 & 2043 & 1089 & 2070 & 183 & 387 \\
\hline 2.5 & 1488 & 2794 & 1496 & 2730 & 1455 & 2796 & 246 & 483 \\
\hline 3.0 & 1759 & 3402 & 1789 & 3317 & 1728 & 3386 & 292 & 592 \\
\hline 3.5 & 2017 & 3843 & 2077 & 3756 & 1996 & 3835 & 341 & 660 \\
\hline 4.0 & 2325 & 4389 & 2383 & 4289 & 2283 & 4379 & 392 & 761 \\
\hline 4.5 & 2685 & 4928 & 2757 & 4816 & 2627 & 4931 & 441 & 845 \\
\hline 5.0 & 2999 & 5525 & 3094 & 5393 & 2926 & 5524 & 487 & 948 \\
\hline 5.5 & 3429 & 6182 & 3519 & 6020 & 3347 & 6181 & 531 & 1024 \\
\hline 6.0 & 3793 & 6742 & 3913 & 6566 & 3741 & 6750 & 601 & 1089 \\
\hline 6.5 & 4108 & 7217 & 4230 & 7036 & 4047 & 7229 & 666 & 1154 \\
\hline 7.0 & 4493 & 7802 & 4605 & 7626 & 4421 & 7815 & 717 & 1221 \\
\hline 7.5 & 4756 & 8279 & 4877 & 8102 & 4685 & 8295 & 762 & 1288 \\
\hline 8.0 & 5079 & 8809 & 5212 & 8619 & 5020 & 8844 & 799 & 1354 \\
\hline 8.5 & 5332 & 9194 & 5460 & 8996 & 5279 & 9220 & 885 & 1424 \\
\hline 9.0 & 5752 & 9851 & 5911 & 9640 & 5708 & 9896 & 913 & 1509 \\
\hline 9.5 & 6181 & $1.043 \mathrm{e}+004$ & 6300 & $1.02 \mathrm{e}+004$ & 6085 & $1.048 \mathrm{e}+004$ & 913 & 1509 \\
\hline 10.0 & 6469 & $1.089 e+004$ & 6587 & $1.066 \mathrm{e}+004$ & 6321 & $1.096 \mathrm{e}+004$ & 1011 & 1625 \\
\hline 10.5 & 6821 & $1.135 \mathrm{e}+004$ & 6931 & $1.112 \mathrm{e}+004$ & 6679 & $1.142 \mathrm{e}+004$ & 1048 & 1674 \\
\hline 11.0 & 7138 & $1.189 \mathrm{e}+004$ & 7256 & $1.164 \mathrm{e}+004$ & 7001 & $1.194 \mathrm{e}+004$ & 1090 & 1744 \\
\hline 11.5 & 7480 & $1.233 e+004$ & 7615 & $1.206 e+004$ & 7351 & $1.238 \mathrm{e}+004$ & 1141 & 1782 \\
\hline 12.0 & 7893 & $1.294 \mathrm{e}+004$ & 8004 & $1.268 \mathrm{e}+004$ & 7729 & $1.302 \mathrm{e}+004$ & 1191 & 1862 \\
\hline 12.5 & 8326 & $1.354 \mathrm{e}+004$ & 8428 & $1.327 \mathrm{e}+004$ & 8140 & $1.361 \mathrm{e}+004$ & 1238 & 1910 \\
\hline 13.0 & 8707 & $1.412 \mathrm{e}+004$ & 8808 & $1.383 e+004$ & 8524 & $1.419 e+004$ & 1295 & 1986 \\
\hline 13.5 & 8977 & $1.463 \mathrm{e}+004$ & 9113 & $1.432 \mathrm{e}+004$ & 8785 & $1.47 \mathrm{e}+004$ & 1342 & 2066 \\
\hline 14.0 & 9386 & $1.507 \mathrm{e}+004$ & 9515 & $1.475 \mathrm{e}+004$ & 9142 & $1.516 \mathrm{e}+004$ & 1405 & 2122 \\
\hline 14.5 & 9803 & $1.569 \mathrm{e}+004$ & 9958 & $1.536 \mathrm{e}+004$ & 9589 & $1.579 \mathrm{e}+004$ & 1466 & 2188 \\
\hline 15.0 & $1.019+004$ & $1.62 e+004$ & $1.036 e+004$ & $1.586 e+004$ & 9959 & $1.629 e+004$ & 1518 & 2249 \\
\hline
\end{tabular}




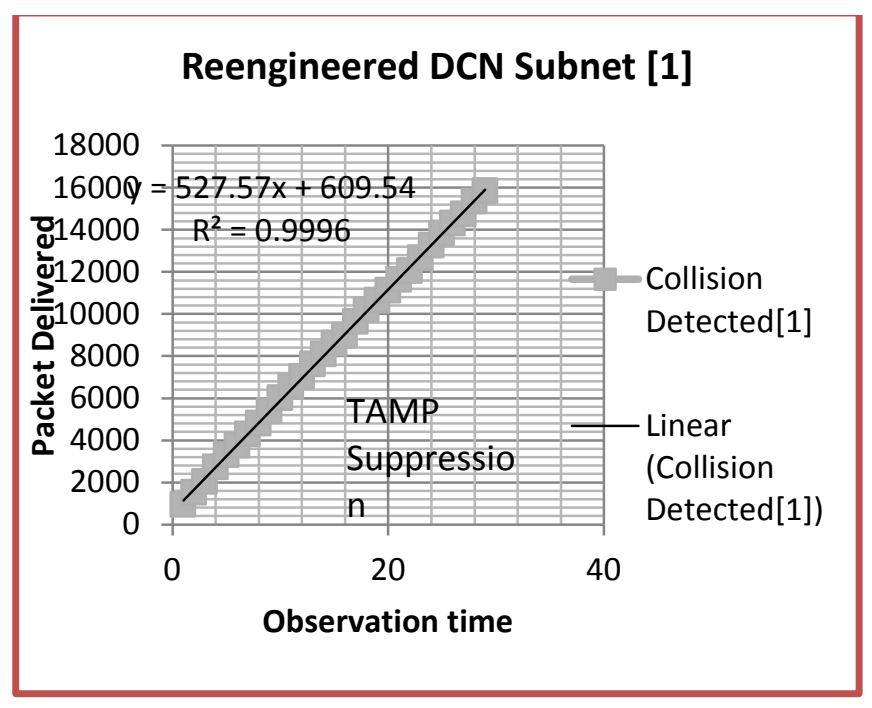

Figure 4. A plot of TAMP Suppression in Subnet [1]

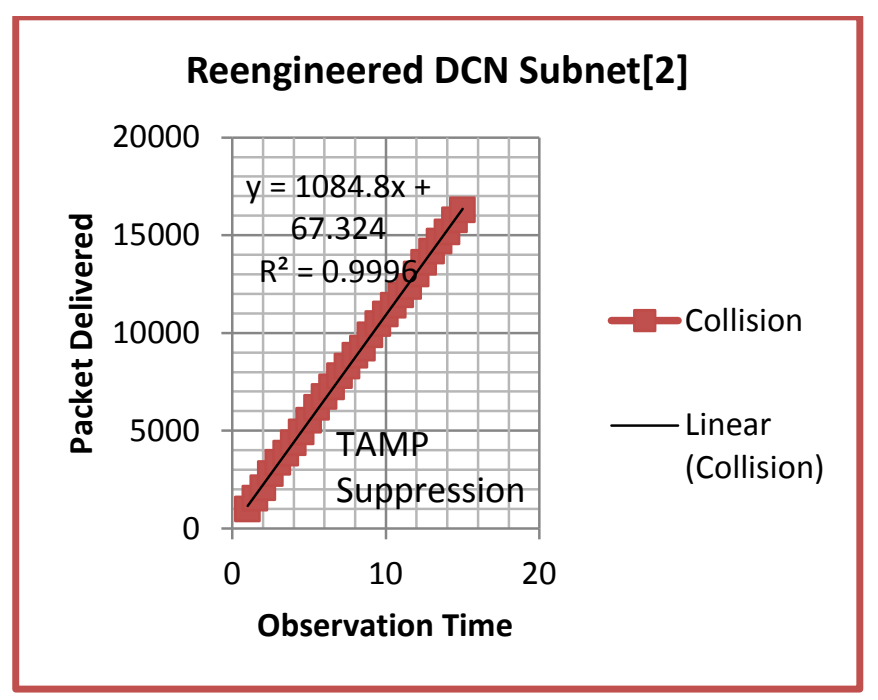

Figure 5. A plot of TAMP Suppression in Subnet [2]

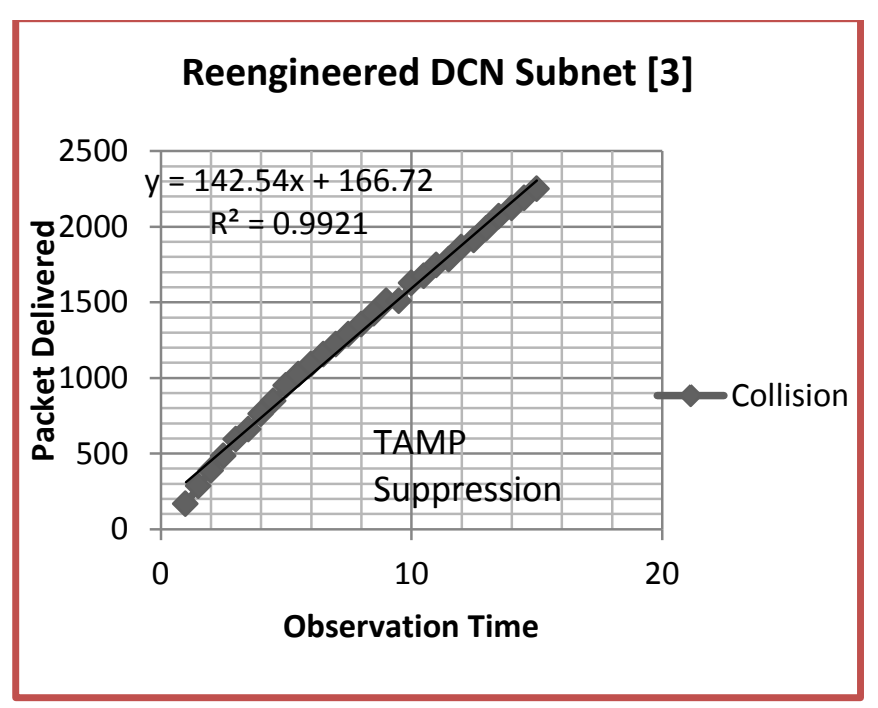

Figure 6. A plot of TAMP Suppression in Subnet [3]

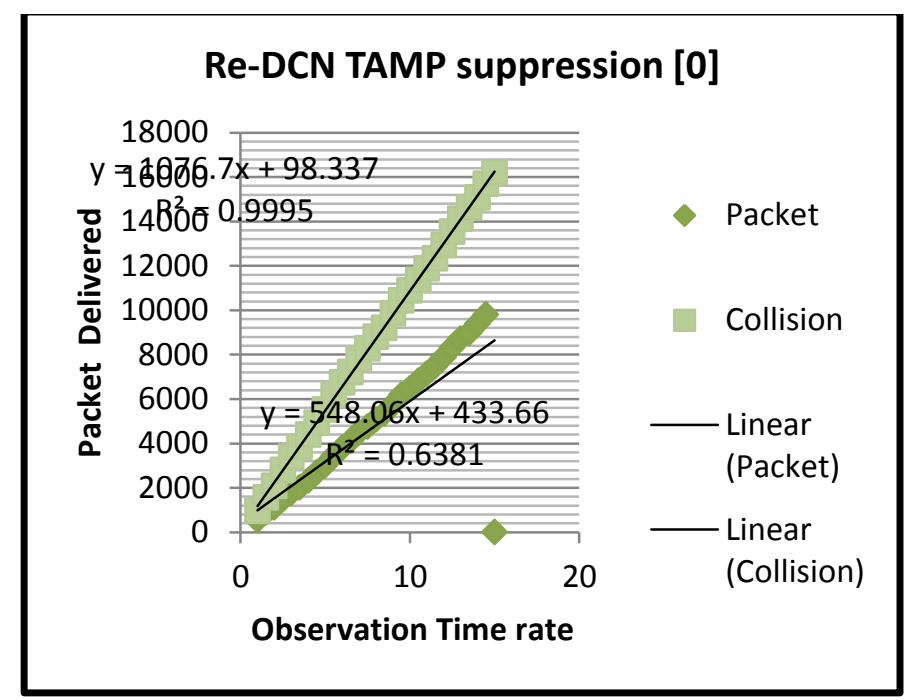

Figure 7. A plot of TAMP Suppression for packet and broadcast traffic

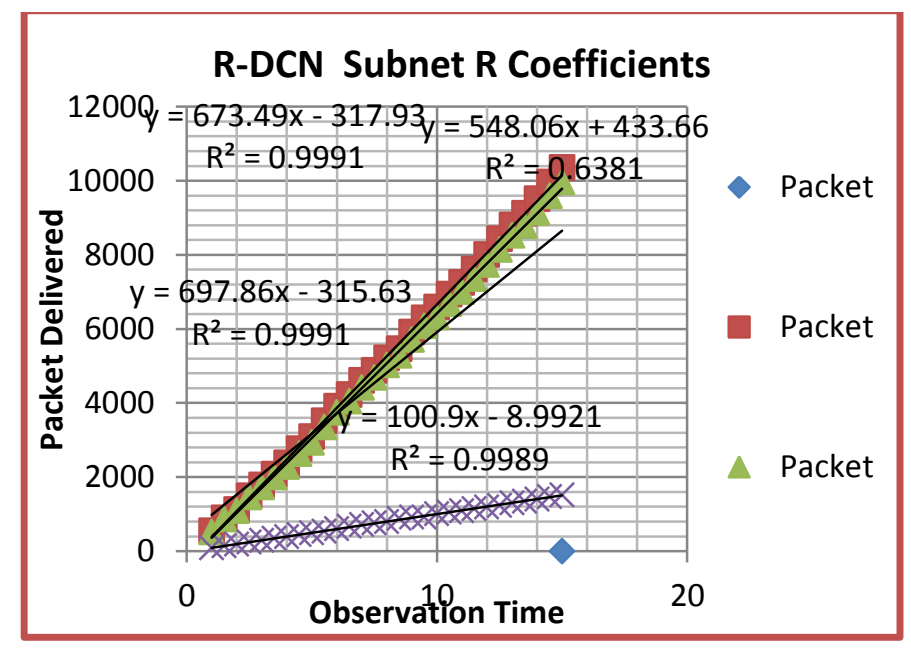

Figure 8. A Plot of Suppression R coefficients for High Scale Model

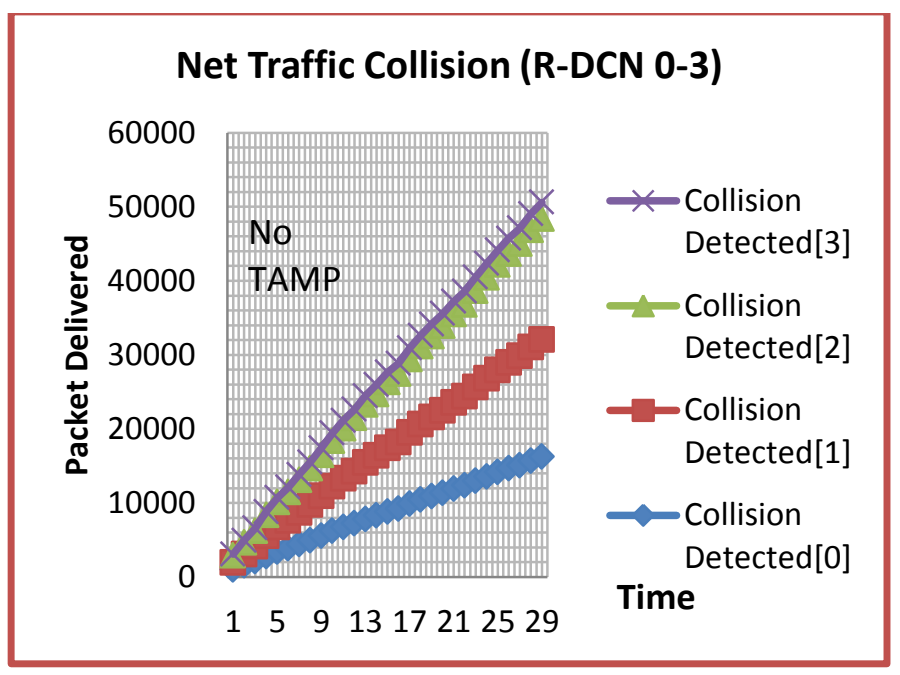

Figure 9. A Plot of Net Collision for subnets 0-3 
From Fig. 7, It is known that packet delivered in a data center network may not be stable at the connection level under some unfair bandwidth allocation policies, even when the normal offered load condition is satisfied, i.e., the average traffic load at each link is less than its capacity. From the plot, under the normal offered load condition in the reengineered DCN, a data network is stable when the bandwidth of the network is allocated so as to maximize a class of general utility functions. In context, packet delivery and collision occurrence are optimized throughout the simulation run time majorly because of the suppression strategy adopted in the model.

Regression analysis as a statistical tool for investigating into performance index and suppression index is used in this work. From Fig. 8, the four $\mathrm{R}$ depicts a positive correlation thereby justifying the model validity. The observation time is the simulation time during which the model statistics are gathered. The packet delivery mode is seen to be reliable, and efficient.

In general, this work assumes that the network should be stable under the normal load condition following the effects of CSMA/CD, but with no TAMP, it shows possible collision cases detected. Though layer 2 switches represent collision domains, yet the full duplex functionality literally does not eradicate collision possibility on the interface ports. For a high end application running on such networks, it is pertinent that all possibilities of collisions be addressed. In this work, Fig. 9 presents the net collision effects for subnet 0-3. As discussed earlier, the approach adopted in this work suppresses interferences and collision possibilities.

Fig. 10 shows a buffer size distribution in a pie chart and presented average throughput. The variation is based on an increment of 50 packets after a previous buffer size selection. This distribution optimally reflects efficient switch traffic carrying capacities with respect to the average throughput in the DCN model. It ranges from 25 packets to 1400 packets for the core switch in the model R-DCN setup. The result of the observation shows that the higher the R-DCN switch buffer, the higher and more effective the throughput with minimal losses. Also, wireless access points with considerable buffer size play significant role in good throughput propagation.

Fig. 11 shows the average network utilization effect for the R-DCN while Fig. 12 shows latency throughput response in the reengineered DCN design. In the context of enterprise DCN, latency, throughput, utilization, and bandwidth usage are vital resources considered in design phases. With the traffic load sources in the hierarchical topology used in this research (Access, Aggregate and Core layers), an initial gradient rise was established before resource allocations were fairly distributed. With a connection request, feasible regions of resource allocation are first established comfortably with CSMA/CD+TAMP. From the test bed, enterprise servers are dumped in Subnet 3 only for server consolidation and virtualization. All the load sources from the model polls resources from this server. Hence, resource utilization in these regions is quite high as shown by the peaks from 6000 and above load times. These are the regions with high VLAN priority tags and as such the computational power of the DCN servers are high. Thus, it was observed that in model, the subnet 3 region have the highest resource utilization cycles. Also, at zero load time from the plot, the connection requests have been established in the model as the buffer capacity of the switches presents a geometric expansion. This literally depicts a high performance network since server virtualization in this region presents a cost effective infrastructure management and maintains high performance traffic workload computing. Hence, the cloud server is hosted in this domain.

According to [15], network throughput is defined as the average rate of successful message delivery over a communication network (access, distribution and core layers in our context). From Fig. 13, the throughput response was seen to be greatly influenced by the CSMA/CD+TAMP. From a packet generation rate of 500 and above, the throughput response was seen to exponentially rise to higher values with very little latency effect, hence depicting a fast convergence network model. It might take a long time for each packet to reach its destination, because it gets held up in long queues, or takes a less direct route to avoid congestion. This is different from throughput, as the delay can build up over time, even if the throughput is almost normal. In some cases, excessive latency can render an application such as VoIP or online gaming unusable. The model in this context shows low latencyhigh throughput responses for all applications on this network.

According to [16], the quality of service (QoS) refers to several related aspects of telephony and computer networks that allow the transport of traffic with special requirements. In particular, traffic delay is a considered metric for multimedia conversations in the reengineered DCN mode, as well as supporting new applications with even more strict service demands. Under the influence of the adopted protocol, after initial startup, at within 4 secs, the network delay collapses and maintains a stable load traffic state. Average delay, in the reengineered DCN model is highly optimized hence giving rise to a stable and faster network model. Essentially, Fig. 13 and Fig. 14 depict typically reengineered traffic characterization. Considering the CSMA/CD+TAMP, this work via its generated data in table $2 \mathrm{a}, 2 \mathrm{~b}$ shows that QoS parameters chosen reflects the desired model behavior.

Fig. 15 shows a fast convergence and effective resource utilization in the proposed model. Application network baseline which is a functional description of application software, how it is used on the proposed network, and the transmission characteristics of the application were all considered in this context. Critical information demanding good utilization and fast convergence include the following: Application server platform, Server location(s), Client locations (number on each LAN) and Application data transmission detail for discrete application transactions.

Conclusively, in all cases, owing to our implementation technologies like virtualization and consolidations, effective service provisioning during the simulation runs shows a high performance reengineered DCN model. 


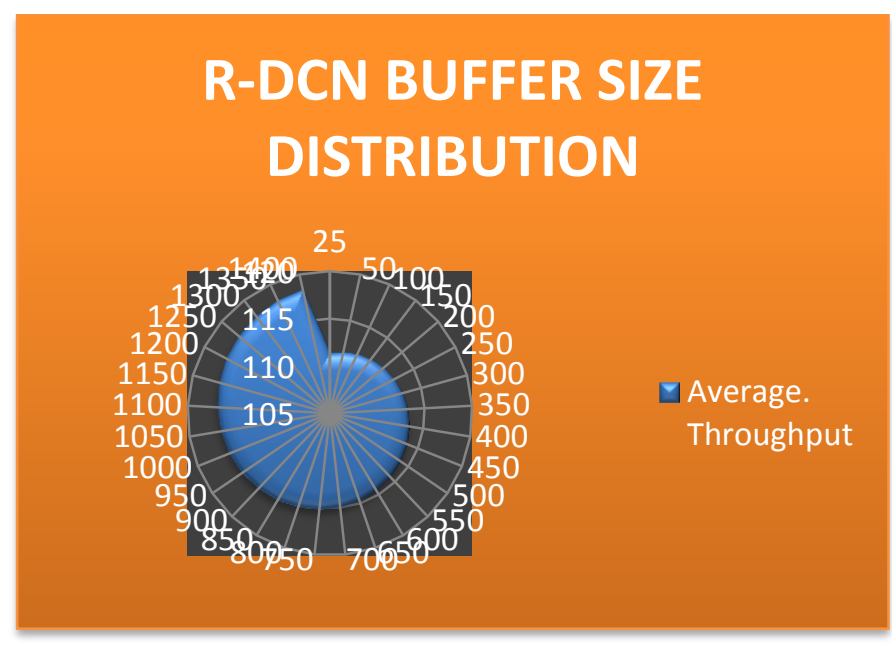

Figure 10. A plot of reengineered DCN switch buffer size distribution

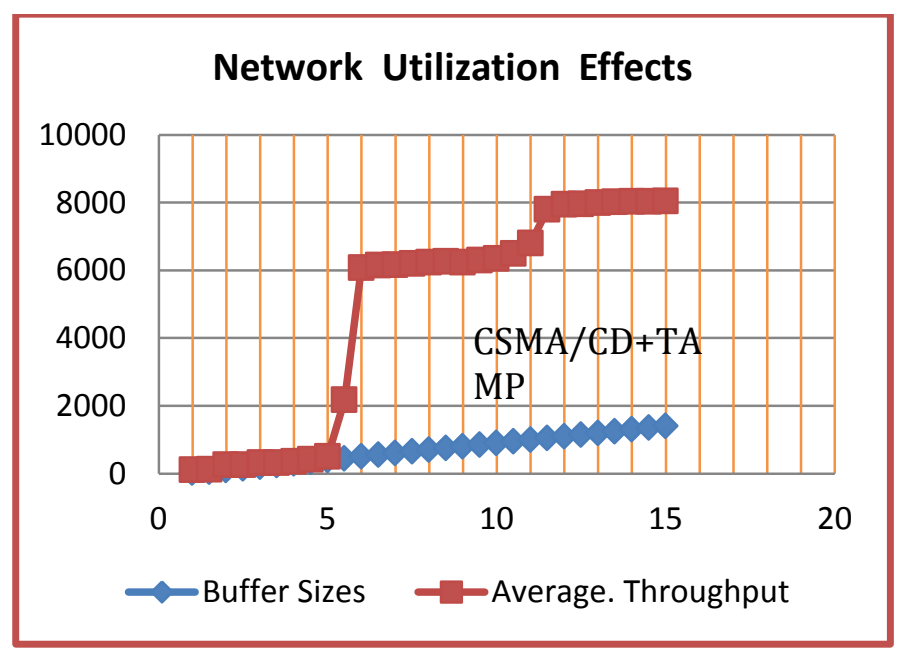

Figure 11. A plot of Reengineered DCN Network Utilization Effects.

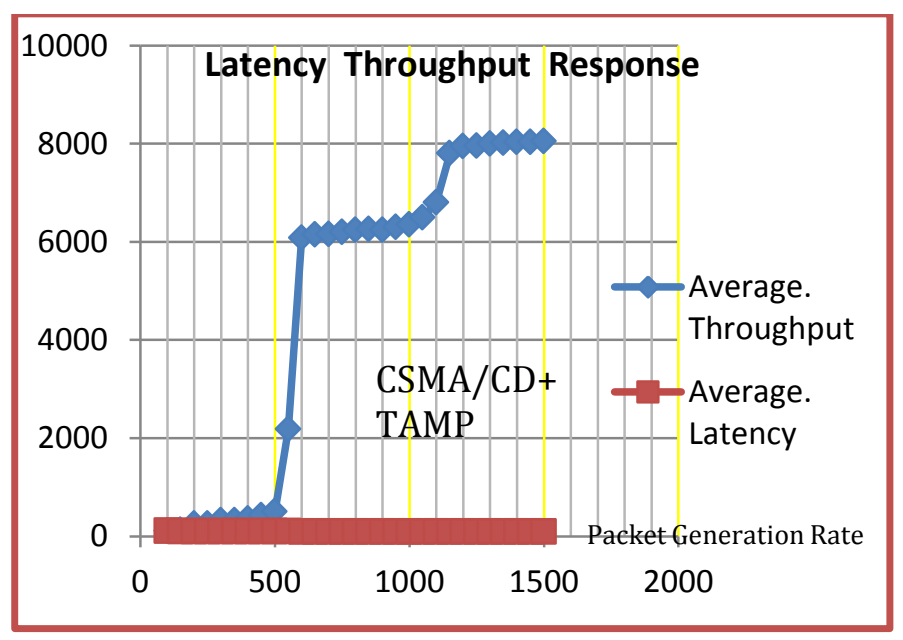

Figure 12. A plot of Reengineered DCN latency Throughput Response

\section{Average Traffic Delay}

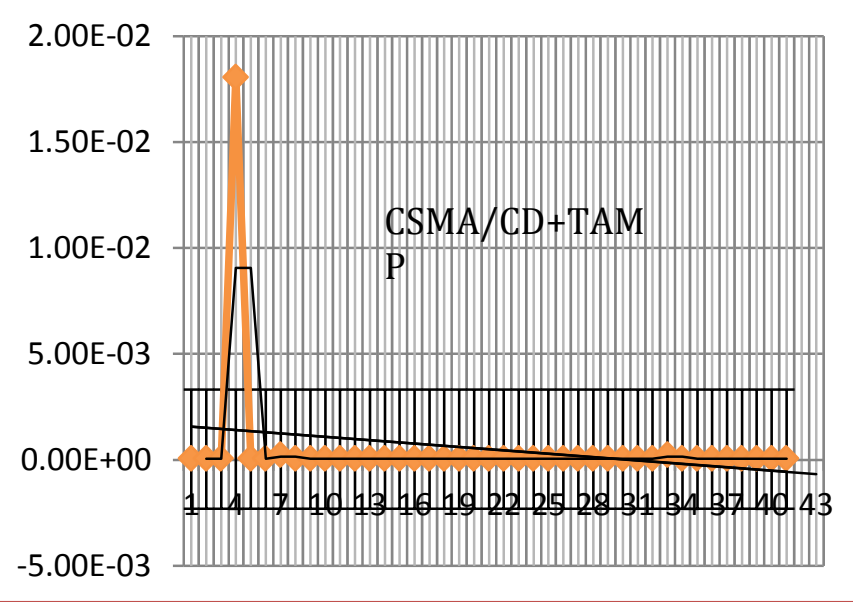

Figure 13. A plot of Reengineered DCN Average Traffic Delay

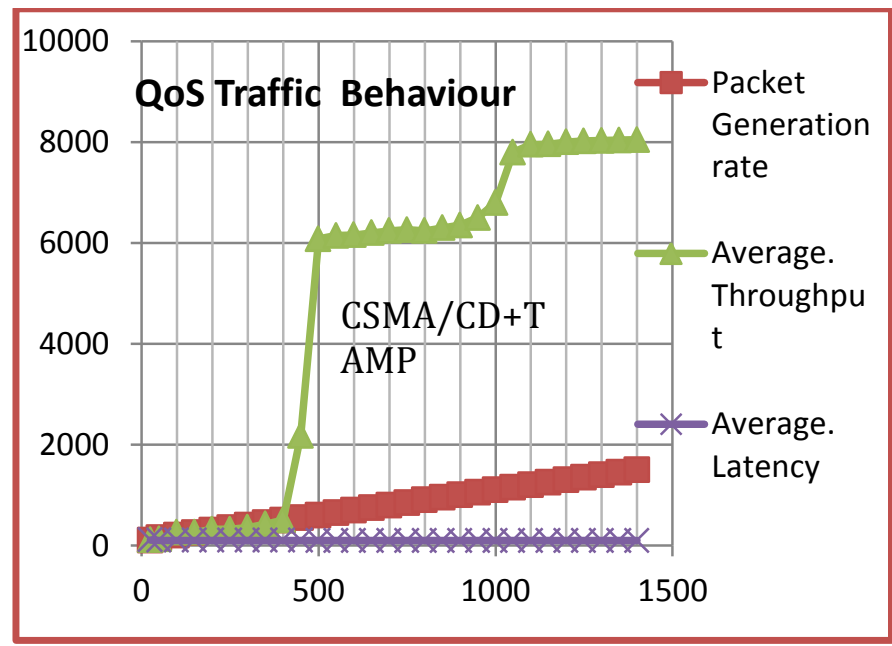

Figure 14. A plot of Reengineered DCN QoS Traffic Behaviour

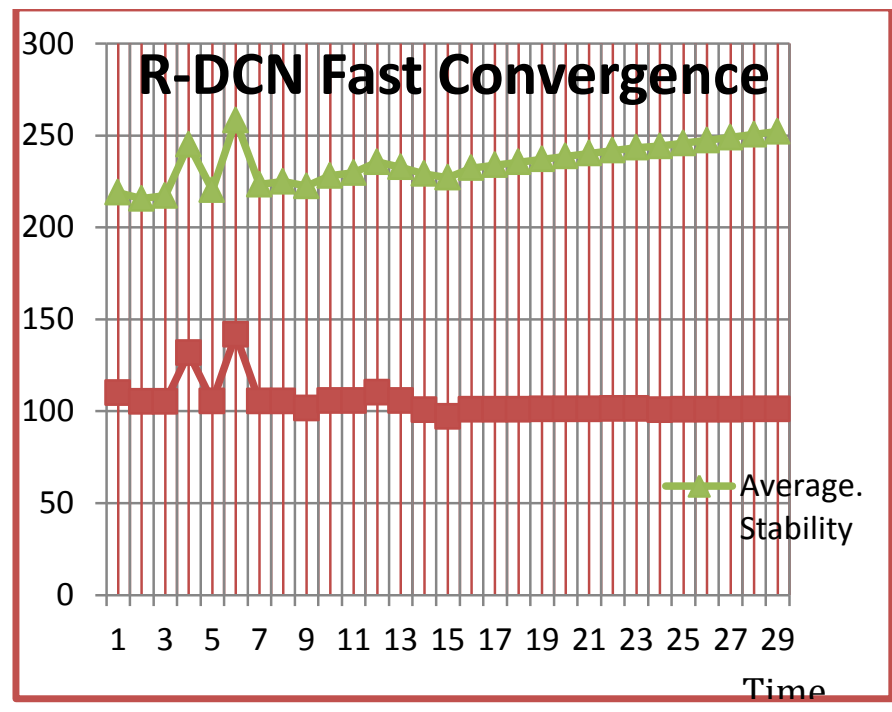

Figure 15. A plot of Reengineered DCN Fast Convergence 


\section{REFERENCES}

[1] Okafor K.C., "SVLAN Approach to Congestion Management in Data Center Networks", M.Eng Thesis, University of Nigeria, Nsukka," 2010.

[2] http://ieee802.org/802_tutorials/Data-Center-Bridging-Tutorial-Nov2007-v2.pdfGigabit Ethernet-Wikipedia.

[3] O. Kennedy, U. Chidiebele. C, C, Prof. H. C. Inyiama, Dr. C.C Okezie, "MATLAB Simevent: A Process Model Approach for Event-Based Communication Network Design, (A Case for Reengineered DCN)" (Unpublished).

[4] ITU-T SG16, "One-way Transmission Time”, Recommendation G.114

[5] ITU-T SG13, "Network Performance Objectives for IP-Based Services", Y.1541

[6] ITU-T SG12, "The E-model, a computational model for use in transmission planning”, Recommendation G.107.

[7] L. Zheng, L. Zhang, D. Xu, "Characteristics of network delay and delay jitter and its effect on voice over IP (VoIP)", ICC 2001, V. 1, June 01 pp.122-126.

[8] Rozalina Dimora, Georgi Georgiev, Zlatko Stanchev. "Performance Analysis of QoS parameters for Voice over IP Applications in a LAN Segment" International Scientific Conference on Computer Science 2008. Pp. 232-237.

[9] Nalin Gahlaut et al. "Comparative Analysis \& Study of Different QoS parameter of wireless AD-HOC Network2". International Journal of Advances in Engineering \& Technology,. Vol. 1 Issue 2, pp. 176-182.

[10] Vijayalaskhmi M, et al "QoS Parameter Analysis on AODV and DSDV Protocols in a wireless Network" International Journal of Communication Network \& Security, Volume1, Issue 1, 2011 pp. 62-70.

[11] I. Miloucleva, A, Nassri, AArizaloni. "Automated Analysis of Network QoS parameters for Voice over IP Applications". IPS 2004, BUDAPEST HUNGARY pp. 184-193.

[12] S. R. Biradar, et al. "Analysis of Qos Parameters for MANETs Routing Protocols" International Journal on Computer Science and Engineering. Vol. 02, No. 03, 2010 pp. 593-599.
[13] Mathworks. 2007. http://www.mathworks.com

[14] http://www.mathworks.in/help/toolbox/simulink/slref/toworkspace.html

[15] http://en.wikipedia.org/wiki/ Throughput

[16] http://en.wikipedia.org/wiki/ Quality_of_service

\section{AUTHORS PROFILE}

Udeze Chidiebele C. received his B.Eng and M.Sc in Electrical Electronics Engineering with Nnamdi Azikwe University, Awka, Nigeria .He is a Senior R \& D Engineer with Electronics development Institute Awka, Nigeria and also a member of NSE. He is currently running his $\mathrm{Ph}$. D programme in computer and control systems engineering. His current research interest is on data center networks, wireless sensor networks and control systems engineering.

Prof H.C Inyiama is a senior lecturer in the electronic and computer department of Nnamdi Azikiwe University Awka, Nigeria. $\mathrm{He}$ is an expert consultant in computer and control systems engineering. He has supervised many masters and Ph.D works in electronics engineering, computer science and engineering, and has many publications to his credit.

Okafor Kennedy. C. is a Senior R \& D Engineer. He has B.Eng \& M.Engr in Digital Electronics \& Computer Engineering from the university of Nigeria Nsukka, (UNN). He a Cisco expert and currently works with the ICT department of Electronics Development Institute, ELDI. He is a member of IEEE, IAENG and NCS.

C.C Okezie is a senior lecturer in the electronic and computer department of Nnamdi Azikiwe University Awka. She received her B. Eng, M. Eng, and Ph.D from Enugu State University of Science and Technology, Enugu, Nigeria. She majors in digital systems and control engineering and has many publications to her credit. 